\title{
Comparison of treatment results between 3- and 2-stage Gamma Knife radiosurgery for large brain metastases: a retrospective multi-institutional study
}

\author{
Toru Serizawa, MD, ${ }^{1}$ Yoshinori Higuchi, MD, ${ }^{2}$ Masaaki Yamamoto, MD, ${ }^{3}$ Shigeo Matsunaga, MD, ${ }^{4}$ \\ Osamu Nagano, MD, ${ }^{5}$ Yasunori Sato, PhD, ${ }^{6}$ Kyoko Aoyagi, MD, ${ }^{5}$ Shoji Yomo, MD, ${ }^{7}$ \\ Takao Koiso, MD, ${ }^{3}$ Toshinori Hasegawa, MD, ${ }^{8}$ Kiyoshi Nakazaki, MD, ${ }^{9}$ Akihito Moriki, MD, ${ }^{10}$ \\ Takeshi Kondoh, MD, ${ }^{11}$ Yasushi Nagatomo, MD, ${ }^{12}$ Hisayo Okamoto, MD, ${ }^{13}$ Yukihiko Kohda, MD, ${ }^{14}$ \\ Hideya Kawai, MD, ${ }^{15}$ Satoka Shidoh, MD, ${ }^{16}$ Toru Shibazaki, MD, ${ }^{17}$ Shinji Onoue, MD, ${ }^{18}$ \\ Hiroyuki Kenai, MD, ${ }^{19}$ Akira Inoue, MD, ${ }^{20}$ and Hisae Mori, MD ${ }^{21}$
}

${ }^{1}$ Tokyo Gamma Unit Center, Tsukiji Neurological Clinic, Tokyo; Departments of ${ }^{2}$ Neurological Surgery and ${ }^{6}$ Global Clinical Research, Chiba University Graduate School of Medicine, Chiba; ${ }^{3}$ Katsuta Hospital Mito GammaHouse, Hitachi-naka; ${ }^{4}$ Stereotactic Radiotherapy Center, Yokohama Rosai Hospital, Yokohama; ${ }^{5} \mathrm{Gamma}$ Knife House, Chiba Cerebral and Cardiovascular Center, Ichihara; 'Division of Radiation Oncology, Aizawa Comprehensive Cancer Center, Aizawa Hospital, Matsumoto; ${ }^{8}$ Department of Neurosurgery, Komaki City Hospital, Komaki; ' $D$ Department of Neurosurgery, Brain Attack Center, Ota Memorial Hospital, Fukuyama; ${ }^{10}$ Kochi Gamma Knife Center, Mominoki Hospital, Kochi; ${ }^{11}$ Department of Neurosurgery, Shinsuma General Hospital, Kobe; ${ }^{12}$ Department of Neurosurgery, Kouseikai Takai Hospital, Tokyo; ${ }^{13}$ Department of Neurosurgery, Takashima Hospital, Yonago; ${ }^{14}$ Department of Neurosurgery, Asanogawa General Hospital, Kanazawa; ${ }^{15}$ Department of Surgical Neurology, Research Institute for Brain and Blood Vessels, Akita; ${ }^{16}$ Department of Neurosurgery, Institute of Brain and Blood Vessels, Mihara Memorial Hospital, Isesaki; ${ }^{17}$ Department of Neurosurgery, Hidaka Hospital, Takasaki; ${ }^{18}$ Department of Neurosurgery, Ehime Prefectural Central Hospital, Matsuyama; ${ }^{19}$ Department of Neurosurgery, Nagatomi Neurosurgical Hospital, Oita; ${ }^{20}$ Department of Neurosurgery, Yamagata Prefectural Central Hospital, Yamagata; and ${ }^{21}$ Department of Neurosurgery, National Cerebral and Cardiovascular Center, Suita, Japan

OBJECTIVE In order to obtain better local tumor control for large (i.e., $>3 \mathrm{~cm}$ in diameter or $>10 \mathrm{~cm}^{3}$ in volume) brain metastases (BMs), 3-stage and 2-stage Gamma Knife surgery (GKS) procedures, rather than a palliative dose of stereotactic radiosurgery, have been proposed. Here, authors conducted a retrospective multi-institutional study to compare treatment results between 3-stage and 2-stage GKS for large BMs.

METHODS This retrospective multi-institutional study involved 335 patients from 19 Gamma Knife facilities in Japan. Major inclusion criteria were 1) newly diagnosed BMs, 2) largest tumor volume of $10.0-33.5 \mathrm{~cm}^{3}, 3$ ) cumulative intracranial tumor volume $\leq 50 \mathrm{~cm}^{3}$, 4) no leptomeningeal dissemination, 5) no more than 10 tumors, and 6) Karnofsky Performance Status $70 \%$ or better. Prescription doses were restricted to between 9.0 and 11.0 Gy in 3-stage GKS and between 11.8 and 14.2 Gy in 2-stage GKS. The total treatment interval had to be within 6 weeks, with at least 12 days between procedures. There were 114 cases in the 3-stage group and 221 in the 2-stage group. Because of the disproportion in patient numbers and the pre-GKS clinical factors between these two GKS groups, a case-matched study was performed using the propensity score matching method. Ultimately, 212 patients (106 from each group) were selected for the case-matched study. Overall survival, tumor progression, neurological death, and radiation-related adverse events were analyzed.

RESULTS In the case-matched cohort, post-GKS median survival time tended to be longer in the 3-stage group (15.9 months) than in the 2-stage group (11.7 months), but the difference was not statistically significant $(p=0.65)$. The cumu-

ABBREVIATIONS BM = brain metastasis; CITV = cumulative intracranial tumor volume; CTCAE = Common Terminology Criteria for Adverse Events; GKS = Gamma Knife surgery; JLGKS = Japanese Leksell Gamma Knife Society; KPS = Karnofsky Performance Status; LQ = linear quadratic; MST = median survival time; NPS = neurological prognostic score; OS = overall survival; RPA = recursive partitioning analysis; SRS = stereotactic radiosurgery; SRT = stereotactic radiotherapy; TKI = tyrosine kinase inhibitor; WBRT = whole-brain radiation therapy.

SUBMITTED October 15, 2017. ACCEPTED April 5, 2018.

INCLUDE WHEN CITING Published online September 7, 2018; DOI: 10.3171/2018.4.JNS172596. 
lative incidences of tumor progression ( $21.6 \%$ vs $16.7 \%$ at 1 year, $p=0.31)$, neurological death $(5.1 \%$ vs $6.0 \%$ at 1 year, $p$ $=0.58)$, or serious radiation-related adverse events (3.0\% vs $4.0 \%$ at 1 year, $p=0.49)$ did not differ significantly.

CONCLUSIONS This retrospective multi-institutional study showed no differences between 3-stage and 2-stage GKS in terms of overall survival, tumor progression, neurological death, and radiation-related adverse events. Both 3-stage and 2-stage GKS performed according to the aforementioned protocols are good treatment options in selected patients with large BMs.

https://thejns.org/doi/abs/10.3171/2018.4.JNS172596

KEYWORDS staged Gamma Knife surgery; large brain metastases; multi-institutional cooperative study; case-matched study; competing risk analysis; oncology; stereotactic radiosurgery

I $\mathrm{N}$ order to improve tumor control for large (i.e., $>3$ $\mathrm{cm}$ in diameter or $>10 \mathrm{~cm}^{3}$ in volume) brain metastases (BMs), Higuchi et al., in 2009, developed a 3-stage Gamma Knife surgery (GKS) procedure using 10-Gy irradiations delivered at 2-week intervals. ${ }^{12}$ However, 3-stage GKS was not widely adopted because of limited support from the public health insurance in Japan, difficulties in schedule adjustments, and the burdens of repeated frame fixation. Since Yomo et al. reported on 2-stage GKS in $2012,{ }^{46}$ nearly half of the institutes in Japan have been using this simpler, more feasible approach. But there has been controversy regarding which is better, 3-stage or 2-stage GKS. Therefore, the Japanese Leksell Gamma Knife Society (JLGKS) conducted a retrospective multi-institutional study to compare treatment results between 3-stage and 2-stage GKS in selected patients harboring large BMs.

\section{Methods}

\section{Patient Population}

This retrospective multi-institutional study (JLGK1601) was approved by the institutional review board (Tsukiji Neurological Clinic IRB) and was registered with the University Medical Information Network (UMIN) Clinical Trial Registry (UMIN000022152). Major eligibility criteria were as follows: 1) newly diagnosed BMs, 2) largest tumor volume of $10.0-33.5 \mathrm{~cm}^{3}, 3$ ) cumulative intracranial tumor volume (CITV) $\left.\leq 50 \mathrm{~cm}^{3}, 4\right)$ no MRI findings of leptomeningeal dissemination, 5) no more than 10 tumors, and 6) Karnofsky Performance Status (KPS) ${ }^{16} 70 \%$ or better. However, patients with a low KPS due to intracranial disease whose neurological symptoms were expected to show improvement after the staged GKS were also included, which was the same as in the JLGK0901 study. ${ }^{4}$ In all cases, a Leksell $\mathrm{G}$ frame (Elekta Instruments) was attached with screw pins with the patient under local anesthesia with or without sedation. Neither the Extend system with Perfexion nor the mask system with ICON was used in this series. No margins were set for the gross, clinical, or planning target volumes. The other radiosurgical techniques were exactly the same as those used in the JLGK0901 study. ${ }^{44}$ The protocol for the present study was also regulated in terms of treatment intervals and prescription doses. The total treatment interval was no more than 42 days, but the procedures had to be at least 12 days apart. Prescription doses had to be 9.0-11.0 Gy in the 3-stage procedure and 11.8-14.2 Gy in the 2-stage GKS. All patients underwent GKS alone, not in combination with concurrent whole-brain radiation therapy (WBRT).

\section{Clinical Outcomes}

The primary endpoint was overall survival (OS), and the secondary endpoints were tumor progression including tumor recurrence and radiation necrosis, neurological deterioration, neurological death, and radiation-related adverse events. Overall survival was defined as the interval between the first GKS procedure and death. Tumor progression was defined as a $20 \%$ increase in the diameter of the enhanced lesion. Tumor progression was divided into tumor recurrence, radiation necrosis, and mixed/undetermined lesions, according to the investigators' judgments, which were based on pathological examination results, clinical course, and various imaging findings on MRI, MR spectroscopy, single-photon emission computed tomography (SPECT), positron emission tomography (PET), etc. ${ }^{3,15,19,34}$ Neurological deterioration was defined as a loss of independent activities of daily living (KPS $60 \%$ or worse) due to intracranial disease progression, i.e., tumor recurrence, leptomeningeal dissemination, cerebral dissemination, progression of untreated intracranial tumors, and other unrelated intracranial disease progression. Systemic deterioration was defined as loss of independent activities of daily living (KPS $60 \%$ or worse) due to extracranial disease progression..$^{31,32}$ Neurological death was defined as death caused by intracranial disease progression, whereas systemic death was attributed to extracranial disease progression. ${ }^{31,32}$ Imaging results were also correlated with observed clinical deterioration as defined by the Common Terminology Criteria for Adverse Events (CTCAE) version 4.03. We recorded radiation-related adverse events as CTCAE grade 3 or worse.

\section{Statistical Analysis}

For the baseline variables, summary statistics were constructed using frequencies and proportions for categorical data and means and standard deviations for continuous variables. We compared patient characteristics using the Fisher exact test for categorical outcomes and t-tests for continuous variables, as appropriate. For the primary endpoint analysis, OS was estimated using the Kaplan-Meier method. For secondary time-to-event outcomes, we introduced a competing risk analysis. ${ }^{8}$ The Fine-Gray generalization makes use of the subdistribution hazard to model cumulative incidence, thereby quantifying the overall benefit or harm of an exposure..$^{8,11}$ We accounted for death as a competing risk for tumor progression, tumor recurrence, and radiation necrosis. Tumor recurrence and radiation necrosis are also considered to be competing risks. Thus, 


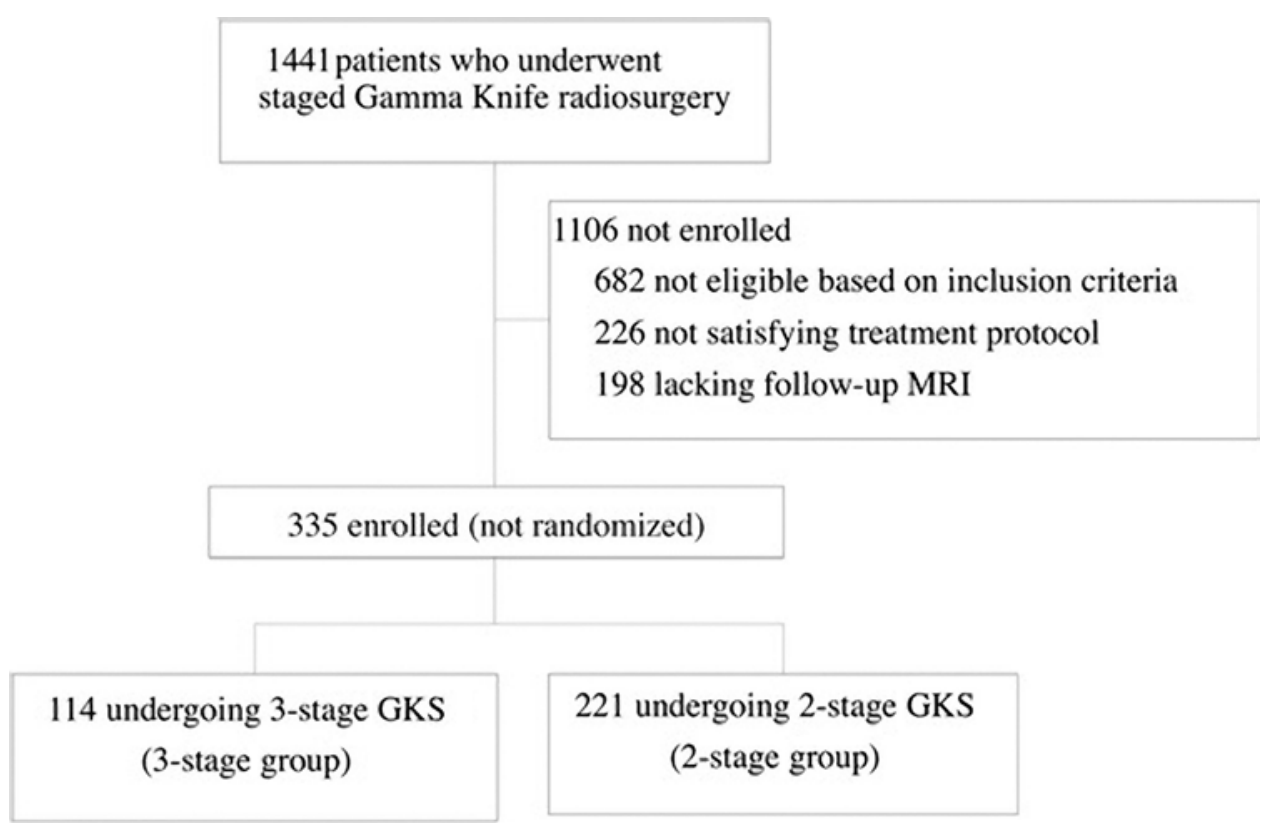

FIG. 1. Trial profile of inclusions and exclusions.

we accounted for "death" as a competing risk for tumor progression, "death or radiation necrosis" as a risk for tumor recurrence, and "death or tumor recurrence" as a risk for radiation necrosis. Mixed/undetermined lesions may have components of both tumor recurrence and radiation necrosis and are counted as failures when estimating the cumulative incidences of both tumor recurrence and radiation necrosis. As a competing risk, we counted "systemic deterioration" as neurological deterioration, ${ }^{31}$ "systemic death" as neurological death, ${ }^{31}$ and "death" as a radiationrelated adverse event.

A case-matched study was also conducted by employing the propensity score matching method with a Greedy 5-to-1 Digit-Match algorithm. ${ }^{27}$ Patient selection was performed for nine clinical factors: age, sex, KPS, primary cancer site, extracranial disease status, neurological symptoms, number of brain tumors, maximum tumor volume, and CITV. All comparisons were planned, and the tests were two-sided. A p value less than 0.05 was considered statistically significant. All statistical analyses were performed by one of the authors (Y.S.), who was not involved in either GKS treatment or patient follow-up and who used SAS software version 9.4 (SAS Institute) and the R statistical program, version 3.0.0.

\section{Results}

\section{Retrospective Study}

Among 1441 cases treated with staged GKS at 19 Gamma Knife sites in Japan, 335 (23.2\%) were eligible for inclusion in this study. Among the 1106 nonregistered patients, 682 were ineligible based on the inclusion criteria, 226 did not satisfy the treatment protocol, and 198 lacked follow-up MRI (Fig. 1). The major reasons the 682 patients did not meet inclusion criteria were as follows: not newly diagnosed BMs (227 patients [33.3\%]), small $\left(<10 \mathrm{~cm}^{3}\right)$ tumor volume $(207$ [30.4\%]), > 10 tumors $(130$ [19.1\%]), large $\left(\geq 33.5 \mathrm{~cm}^{3}\right)$ tumor volume $(106[15.5 \%])$, large $(\geq 50$ $\left.\mathrm{cm}^{3}\right)$ CITV (50 [7.3\%]), low (<70\%) KPS (50 [7.3\%]), and the presence of leptomeningeal dissemination (41 [6.0\%]).

There were 114 cases in the 3 -stage GKS group and 221 in the 2-stage group. During the treatment period from the first to the last staged GKS procedures, 485 lesions were treated with staged GKS (336 lesions in the 2-stage group and 149 in the 3-stage group) and 443 with simultaneous single-session GKS. The median follow-up time after the first GKS procedure for censored observations (101 cases) was 11.7 months (range 2.9-74.3 months), and 234 patients (69.9\%) had died. Among the 275 cases (102 vs 173 in the 3 -stage and 2-stage groups, respectively) with neurological symptoms at the first staged GKS, improvement at the last staged GKS procedure was seen in $168(61.1 \%$; 50 [49.0\%] vs 118 [68.2\%]), no change in $99(36.0 \% ; 50$ [49.0\%] vs $49[28.3 \%])$, and deterioration in $8(2.9 \% ; 2$ [2.0\%] vs $6[3.5 \%])$. Among the 265 patients who required steroid administration at the first staged GKS procedure, steroid doses were maintained or increased at the time of the last staged GKS procedure and 3 months later, respectively, in $116(43.8 \%)$ and $73(27.5 \%)$, decreased in 117 $(44.2 \%)$ and $33(12.5 \%)$, and discontinued in $32(12.1 \%)$ and $159(60.0 \%)$.

The overall median survival time (MST) after the first GKS procedure for the 335 cases was 12.3 months $(95 \%$ CI 10.6-15.0 months). Among the 234 deceased cases, deaths were systemic in $190(81.2 \%)$ and neurological in $44(18.8 \%)$. The causes of neurological death were classified as leptomeningeal dissemination in 21 cases $(47.7 \%)$, recurrence of the GKS-treated lesions in 11 (25.0\%), tumor bleeding in $2(4.5 \%)$, radiation necrosis in $2(4.5 \%)$, cerebral dissemination in $1(2.3 \%)$, growth of untreated lesions in $1(2.3 \%)$, and other/undetermined in $6(13.6 \%)$. For the 86 cases with tumor progression, the diagnosis 
TABLE 1. Clinical characteristics of main cohort of 335 patients and the staged GKS groups

\begin{tabular}{|c|c|c|c|c|}
\hline Category & Entire Cohort & 3-Stage GKS & 2-Stage GKS & p Value* \\
\hline No. of patients & 335 & 114 & 221 & \\
\hline Mean age in yrs (SD) & $66.1(11.7)$ & $65.7(11.4)$ & $66.4(11.8)$ & 0.63 \\
\hline Female sex, no. (\%) & $136(40.6 \%)$ & $90(40.7 \%)$ & $46(40.4 \%)$ & 1.00 \\
\hline KPS $\geq 80 \%$, no. $(\%)$ & $224(66.9 \%)$ & $74(64.9 \%)$ & $150(67.9 \%)$ & 0.63 \\
\hline Stable extracranial disease, no. (\%) & $50(14.9 \%)$ & $9(7.9 \%)$ & $41(18.6 \%)$ & 0.009 \\
\hline \multicolumn{5}{|l|}{ Primary cancer, no. (\%) } \\
\hline NSCL & $141(42.1 \%)$ & $46(40.4 \%)$ & $95(43.0 \%)$ & $0.49 \dagger$ \\
\hline SCL & $21(6.3 \%)$ & $6(5.3 \%)$ & $15(6.8 \%)$ & \\
\hline GIT & $75(22.4 \%)$ & $26(22.8 \%)$ & $49(22.2 \%)$ & \\
\hline Breast & $59(17.6 \%)$ & $20(17.5 \%)$ & $39(17.6 \%)$ & \\
\hline Urogenital & $33(9.9 \%)$ & $15(13.2 \%)$ & $18(8.1 \%)$ & \\
\hline Other & $6(1.8 \%)$ & $1(0.9 \%)$ & $5(2.3 \%)$ & \\
\hline \multicolumn{5}{|l|}{ Modified RPA class, no. (\%) } \\
\hline $1+\| \mathrm{a}$ & $56(16.7 \%)$ & $10(8.8 \%)$ & $46(20.8 \%)$ & 0.016 \\
\hline $\mathrm{Ilb}$ & $78(23.3 \%)$ & $30(26.3 \%)$ & $48(21.7 \%)$ & \\
\hline$\|c+\|$ & $201(60.0 \%)$ & $74(64.9 \%)$ & $127(57.5 \%)$ & \\
\hline Neurological symptoms at 1st staged GKS, no. (\%) & $275(82.1 \%)$ & $102(89.5 \%)$ & $173(78.3 \%)$ & 0.011 \\
\hline Mean no. of brain tumors (SD) & $2.59(2.04)$ & $2.58(2.01)$ & $2.59(2.06)$ & 0.95 \\
\hline Mean max tumor vol in $\mathrm{cm}^{3}(\mathrm{SD})$ & $17.9(6.23)$ & $19.4(6.65)$ & $17.1(5.86)$ & 0.001 \\
\hline Mean CITV in $\mathrm{cm}^{3}$ (SD) & $20.7(8.22)$ & $21.9(8.18)$ & $20.0(8.19)$ & 0.047 \\
\hline
\end{tabular}

$\mathrm{GIT}=$ gastrointestinal; $\mathrm{NSCL}=$ non-small cell lung; $\mathrm{SCL}=$ small cell lung.

${ }^{*}$ The Student t-test was used for continuous variables and the Fisher exact test for pairs of categorical variables.

† Lung versus non-lung.

was tumor recurrence in $58(67.4 \%)$, radiation necrosis in $21(24.4 \%)$, and mixed/undetermined lesions in $7(8.1 \%)$. Management among the 58 cases with tumor recurrence consisted of re-irradiation (42 [72.4\%]), surgical removal (11 [19.0\%]), and observation including medication (5 [8.6\%]). For the 21 cases of radiation necrosis, post-GKS management included medication in 9 (42.9\%; all receiving steroids, and none receiving bevacizumab), observation in $6(28.6 \%)$, and surgical removal in $6(28.6 \%)$. Of the 7 cases with mixed/undetermined lesions, 4 (57.1\%) underwent re-irradiation, 2 (28.6\%) surgical removal, and $1(14.3 \%)$ observation.

Clinical characteristics are compared between 3-stage and 2 -stage groups in Table 1 . In the 3 -stage group, modified recursive partitioning analysis (RPA) ${ }^{43}$ classes were significantly poorer, extracranial disease status more active, neurological symptoms more frequent, tumor volumes larger, and CITV larger. The other clinical factors were similar except for the staged GKS treatment parameters. There were no significant differences in OS, tumor progression including tumor recurrence and radiation necrosis, neurological deterioration, neurological death, or radiation-related adverse events (Table 2 ).

\section{Case-Matched Study}

Propensity score matches considered nine clinical factors, and 212 patients (106 in each group) were ultimately selected. Baseline characteristics were compared between the two staged GKS groups (Table 3). The p values for all matched clinical factors were over 0.32 . Treatment param- eters for staged GKS in the main cohort of 335 patients and the 212 case-matched patients are summarized in Table 4. Treatment outcomes were compared between the two GKS groups for the case-matched patients in Table 5. Although the post-GKS MST was shorter in the 2-stage group (11.7 months) than in the 3 -stage group (15.9 months; Fig. 2), this difference was not statistically significant (HR $1.080,95 \%$ CI $0.777-1.502, \mathrm{p}=0.65)$. Cumulative incidences of tumor progression were slightly higher in the 3 -stage group than in the 2 -stage group $(21.6 \%$ vs $16.7 \%$ at 1 year, respectively; Fig. 3), but these differences did not reach statistical significance $(\mathrm{p}=0.31)$. Tumor recurrence tended to be more frequent in the 3 -stage group, but again the difference was not significant ( $p=0.19$; Table 5). Incidences of radiation necrosis were essentially the same $(p$ $=0.79$ ). Cumulative incidences of neurological deterioration were slightly higher in the 3 -stage group than in the 2 -stage group (16.4\% vs $12.0 \%$ at 1 year, respectively), but these differences did not reach statistical significance $(\mathrm{p}$ $=0.08)$. Neurological death incidences were essentially the same $(5.1 \%$ vs $6.0 \%$ at 1 year, respectively, $\mathrm{p}=0.58)$. The cumulative incidences of CTCAE grade 3 or worse at 1 year were $3.0 \%$ in the 3 -stage group and $4.0 \%$ in the 2 -stage group, and the difference between the two groups was not significant $(\mathrm{p}=0.49)$.

\section{Discussion}

\section{Staged GKS for Large BMs}

Prognosis in patients with large BMs is generally poor 
TABLE 2. Treatment outcomes for the main cohort of 335 patients

\begin{tabular}{|c|c|c|c|c|c|c|}
\hline \multirow[b]{2}{*}{ Variable } & \multicolumn{4}{|c|}{ Post-GKS Yr (cumulative incidence) } & \multirow[b]{2}{*}{$\mathrm{HR}(95 \% \mathrm{Cl})$} & \multirow[b]{2}{*}{ p Value } \\
\hline & 0.5 & 1 & 2 & 3 & & \\
\hline \multicolumn{7}{|l|}{ OS } \\
\hline 3-stage group & 0.751 & 0.546 & 0.361 & 0.200 & 1.169 & 0.26 \\
\hline 2-stage group & 0.720 & 0.505 & 0.246 & 0.158 & $0.89-1.536$ & \\
\hline \multicolumn{7}{|l|}{ Tumor progression* } \\
\hline 3 -stage group & 0.102 & 0.210 & 0.286 & 0.334 & 0.880 & 0.56 \\
\hline 2-stage group & 0.135 & 0.207 & 0.283 & 0.283 & $0.575-1.348$ & \\
\hline \multicolumn{7}{|l|}{ Tumor recurrence ${ }^{*}$} \\
\hline 3-stage group & 0.074 & 0.159 & 0.224 & 0.272 & 0.834 & 0.47 \\
\hline 2-stage group & 0.103 & 0.163 & 0.217 & 0.217 & $0.511-1.359$ & \\
\hline \multicolumn{7}{|l|}{ Radiation necrosis* } \\
\hline 3-stage group & 0.027 & 0.038 & 0.038 & 0.057 & 0.927 & 0.85 \\
\hline 2-stage group & 0.024 & 0.036 & 0.068 & 0.068 & $0.430-1.998$ & \\
\hline \multicolumn{7}{|c|}{ Neurological deterioration } \\
\hline 3-stage group & 0.100 & 0.152 & 0.203 & 0.280 & 0.901 & 0.67 \\
\hline 2-stage group & 0.101 & 0.153 & 0.238 & 0.282 & $0.581-1.417$ & \\
\hline \multicolumn{7}{|l|}{ Neurological death } \\
\hline 3 -stage group & 0.037 & 0.048 & 0.086 & 0.133 & 1.147 & 0.67 \\
\hline 2-stage group & 0.037 & 0.068 & 0.150 & 0.158 & $0.615-2.137$ & \\
\hline \multicolumn{7}{|c|}{ Radiation-related adverse events $\dagger$} \\
\hline 3-stage group & 0.027 & 0.037 & 0.037 & 0.053 & 1.061 & 0.90 \\
\hline 2-stage group & 0.023 & 0.035 & 0.056 & 0.080 & $0.401-2.808$ & \\
\hline
\end{tabular}

* The incidences of tumor progression are not the sum of those for tumor recurrence and radiation necrosis because mixed/undetermined lesions are counted as both tumor recurrence and radiation necrosis.

† CTCAE grade 3 or worse.

TABLE 3. Clinical characteristics of two GKS groups in the 212 case-matched patients

\begin{tabular}{|c|c|c|c|c|}
\hline Category & Total & 3-Stage GKS & 2-Stage GKS & p Value* \\
\hline No. of patients & 212 & 106 & 106 & \\
\hline Mean age in yrs (SD) & $66.1(11.7)$ & $66.3(11.4)$ & $65.9(12.0)$ & 0.80 \\
\hline Female sex, no. (\%) & $86(40.6 \%)$ & $42(39.6 \%)$ & $44(41.5 \%)$ & 0.89 \\
\hline KPS $\geq 80 \%$, no. $(\%)$ & $139(65.6 \%)$ & $69(65.1 \%)$ & $70(66.0 \%)$ & 1.00 \\
\hline Stable extracranial disease, no. (\%) & $17(8.0 \%)$ & $9(8.5 \%)$ & $8(7.5 \%)$ & 1.00 \\
\hline \multicolumn{5}{|l|}{ Primary cancer, no. (\%) } \\
\hline NSCL & $79(37.3 \%)$ & $42(39.6 \%)$ & $37(34.9 \%)$ & $0.58 \dagger$ \\
\hline SCL & $12(5.7 \%)$ & $6(5.7 \%)$ & $6(5.7 \%)$ & \\
\hline GIT & $50(23.6 \%)$ & $24(22.6 \%)$ & $26(24.5 \%)$ & \\
\hline Breast & $42(19.8 \%)$ & $20(18.9 \%)$ & $22(20.8 \%)$ & \\
\hline Urogenital & $27(12.7 \%)$ & $13(12.3 \%)$ & $14(13.2 \%)$ & \\
\hline Other & $2(0.9 \%)$ & $1(0.9 \%)$ & $1(0.9 \%)$ & \\
\hline \multicolumn{5}{|l|}{ Modified RPA class } \\
\hline $1+\| l a$ & $22(10.4 \%)$ & $10(9.4 \%)$ & $12(11.3 \%)$ & 0.83 \\
\hline $\mathrm{Ilb}$ & $53(25.0 \%)$ & $28(26.4 \%)$ & $25(23.6 \%)$ & \\
\hline$\|c+\|$ & $137(64.6 \%)$ & $68(64.2 \%)$ & $69(65.1 \%)$ & \\
\hline Neurological symptoms at 1 st staged GKS, no. (\%) & $182(85.8 \%)$ & $94(88.7 \%)$ & $88(83.0 \%)$ & 0.33 \\
\hline Mean no. of brain tumors (SD) & $2.6(1.9)$ & $2.6(2.1)$ & $2.5(1.8)$ & 0.60 \\
\hline Mean max tumor vol in $\mathrm{cm}^{3}$ (SD) & $18.6(6.5)$ & $18.8(6.5)$ & $18.4(6.6)$ & 0.58 \\
\hline CITV in $\mathrm{cm}^{3}(\mathrm{SD})$ & $21.1(8.2)$ & $21.4(8.2)$ & $20.8(8.3)$ & 0.60 \\
\hline
\end{tabular}

* The Student t-test was used for continuous variables and the Fisher exact test for pairs of categorical variables.

† Lung versus non-lung. 
TABLE 4. Treatment parameters for staged GKS in the main cohort of 335 patients and the 212 case-matched patients

\begin{tabular}{|c|c|c|c|c|c|c|}
\hline \multirow[b]{2}{*}{ Variable } & \multicolumn{3}{|c|}{ Main Cohort } & \multicolumn{3}{|c|}{ Case-Matched Patients } \\
\hline & 3-Stage GKS & 2-Stage GKS & $p$ Value & 3-Stage GKS & 2-Stage GKS & $\mathrm{p}$ Value \\
\hline No. of cases & 114 & 221 & & 106 & 106 & \\
\hline Mean prescription dose in Gy (SD)* & $10.0(0.2)$ & $13.2(0.6)$ & $<0.001$ & $10.0(0.20)$ & $13.2(0.6)$ & $<0.001$ \\
\hline Mean prescription \% (SD) & $50.0(1.8)$ & $48.8(2.7)$ & 0.001 & $50.0(1.9)$ & $48.7(3.5)$ & 0.002 \\
\hline Mean max dose in Gy (SD) & $20.0(0.9)$ & $27.2(2.7)$ & $<0.001$ & $20.0(0.9)$ & $27.3(2.8)$ & 0.002 \\
\hline Mean treatment interval in days (SD)† & $28.6(2.4)$ & $21.4(6.8)$ & $<0.001$ & $28.8(2.3)$ & $22.5(7.2)$ & $<0.001$ \\
\hline Mean initial tumor vol in $\mathrm{cm}^{3}(\mathrm{SD}) \ddagger$ & $19.4(6.7)$ & $17.1(5.9)$ & 0.001 & $18.8(6.5)$ & $18.4(6.5)$ & 0.58 \\
\hline Mean last tumor vol in $\mathrm{cm}^{3}(\mathrm{SD}) \mp \S$ & $12.0(7.3)$ & $11.7(6.7)$ & 0.70 & $11.6(6.9)$ & $12.2(6.9)$ & 0.58 \\
\hline Mean tumor vol reduction ratio (SD) $\ddagger$ & $37.4(33.9)$ & $31.7(31.4)$ & $<0.001$ & $37.4(34.3)$ & $33.5(30.4)$ & 0.38 \\
\hline
\end{tabular}

if good tumor control cannot be attained. Local control failure leads to eventual neurological deterioration and death. For patients with a good performance status and longer expected survival, it is very important to achieve better local control. Total removal of a large BM with or without WBRT is, without question, ideal, regardless of the BM size, if the tumor is surgically accessible and single. ${ }^{28-30}$ Fractionated stereotactic radiotherapy (SRT) is also a good treatment option if the number of tumors is limited..$^{6,20,22,25,41}$ In contrast, for patients in a relatively poor condition with large tumors, palliative stereotactic radiosurgery (SRS) or WBRT is available.? Tumor progression incidence at 1 year for large BMs treated with palliative SRS reportedly ranges from $35 \%$ to $60 \% .^{2,4,24,33}$ For these large tumors, Higuchi et al., in 2009, first described 3-stage GKS to obtain better tumor control than palliative SRS or hypofractionated SRT. ${ }^{12}$ Three-stage GKS using 10-Gy irradiations at each session, with a 2 -week interval between sessions, was revolutionary in terms of tumor volume reduction, in comparison to that obtained with conventional

TABLE 5. Treatment outcomes for the 212 case-matched patients

\begin{tabular}{|c|c|c|c|c|c|c|}
\hline \multirow[b]{2}{*}{ Variable } & \multicolumn{4}{|c|}{ Post-GKS Yr (cumulative incidence) } & \multirow[b]{2}{*}{$\operatorname{HR}(95 \% \mathrm{Cl})$} & \multirow[b]{2}{*}{ p Value } \\
\hline & 0.5 & 1 & 2 & 3 & & \\
\hline \multicolumn{7}{|l|}{ OS } \\
\hline 3 -stage group & 0.772 & 0.574 & 0.378 & 0.210 & $1.080(0.777-1.502)$ & 0.65 \\
\hline 2-stage group & 0.689 & 0.492 & 0.338 & 0.237 & & \\
\hline \multicolumn{7}{|l|}{ Tumor progression* } \\
\hline 3-stage group & 0.101 & 0.216 & 0.296 & 0.345 & $0.763(0.452-1.286)$ & 0.31 \\
\hline 2-stage group & 0.087 & 0.167 & 0.266 & 0.266 & & \\
\hline \multicolumn{7}{|l|}{ Tumor recurrence* } \\
\hline 3-stage group & 0.070 & 0.161 & 0.229 & 0.178 & $0.658(0.352-1.229)$ & 0.19 \\
\hline 2-stage group & 0.049 & 0.116 & 0.198 & 0.198 & & \\
\hline \multicolumn{7}{|l|}{ Radiation necrosis* } \\
\hline 3-stage group & 0.040 & 0.065 & 0.077 & 0.077 & $1.130(0.464-2.752)$ & 0.79 \\
\hline 2-stage group & 0.038 & 0.051 & 0.100 & 0.100 & & \\
\hline \multicolumn{7}{|c|}{ Neurological deterioration } \\
\hline 3-stage group & 0.107 & 0.164 & 0.216 & 0.297 & $0.592(0.331-1.058)$ & 0.078 \\
\hline 2-stage group & 0.076 & 0.120 & 0.177 & 0.197 & & \\
\hline \multicolumn{7}{|l|}{ Neurological death } \\
\hline 3 -stage group & 0.040 & 0.051 & 0.091 & 0.140 & $0.803(0.369-1.745)$ & 0.58 \\
\hline 2-stage group & 0.029 & 0.060 & 0.106 & 0.132 & & \\
\hline \multicolumn{7}{|c|}{ Radiation-related adverse events $\dagger$} \\
\hline 3-stage group & 0.019 & 0.030 & 0.030 & 0.047 & $1.482(0.479-4.588)$ & 0.49 \\
\hline 2-stage group & 0.029 & 0.040 & 0.057 & 0.079 & & \\
\hline
\end{tabular}

* The incidences of tumor progression are not the sum of those for tumor recurrence and radiation necrosis because mixed/undetermined lesions are counted as both tumor recurrence and radiation necrosis.

† CTCAE grade 3 or worse. 


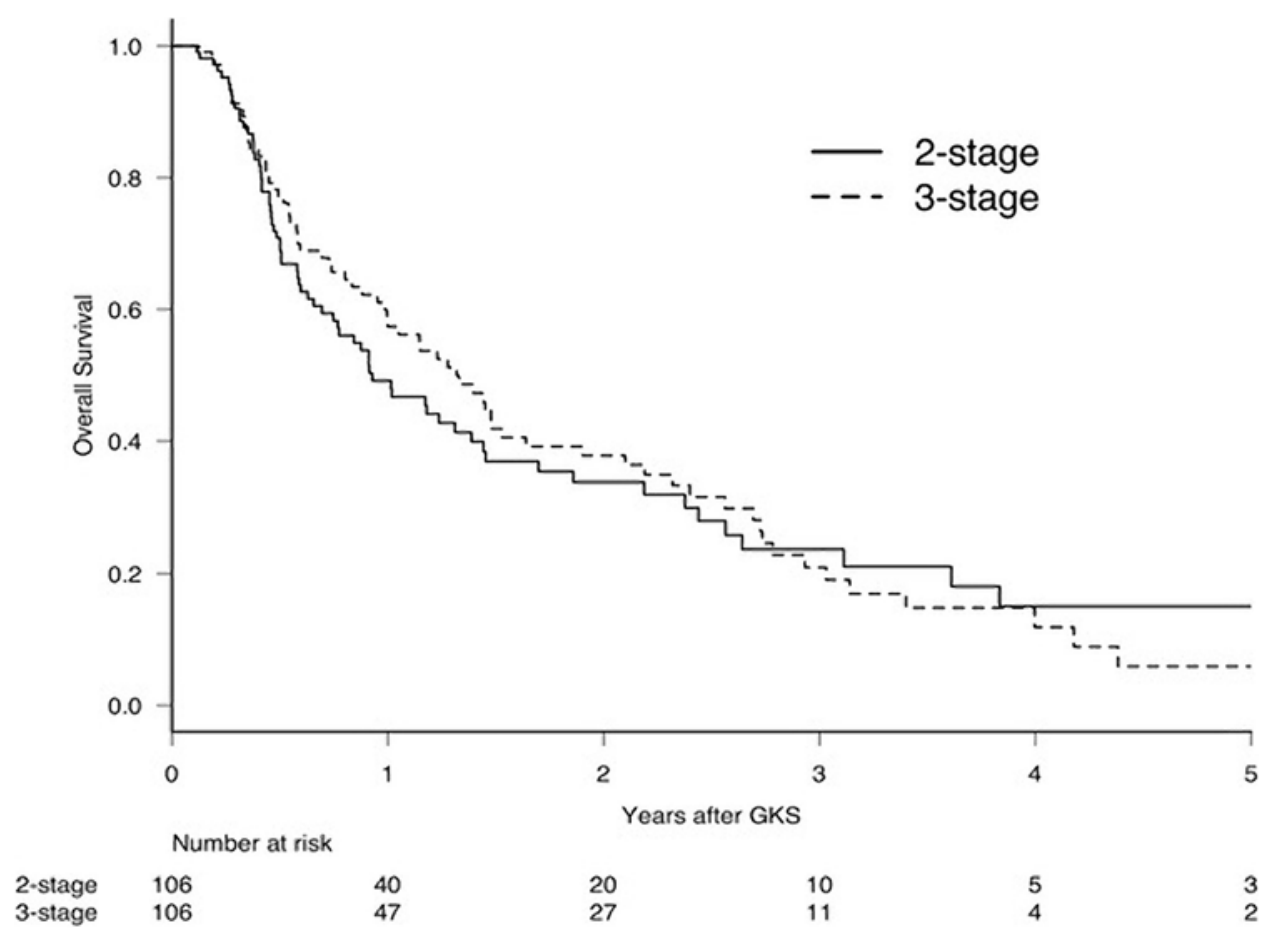

FIG. 2. Overall survival: 3 -stage versus 2 -stage GKS in the 212 case-matched patients. Although the post-GKS MST was shorter in the 2-stage group (11.7 months) than in the 3-stage group (15.9 months), this difference was not statistically significant (HR $1.080,95 \% \mathrm{Cl} 0.777-1.502, \mathrm{p}=0.65)$.

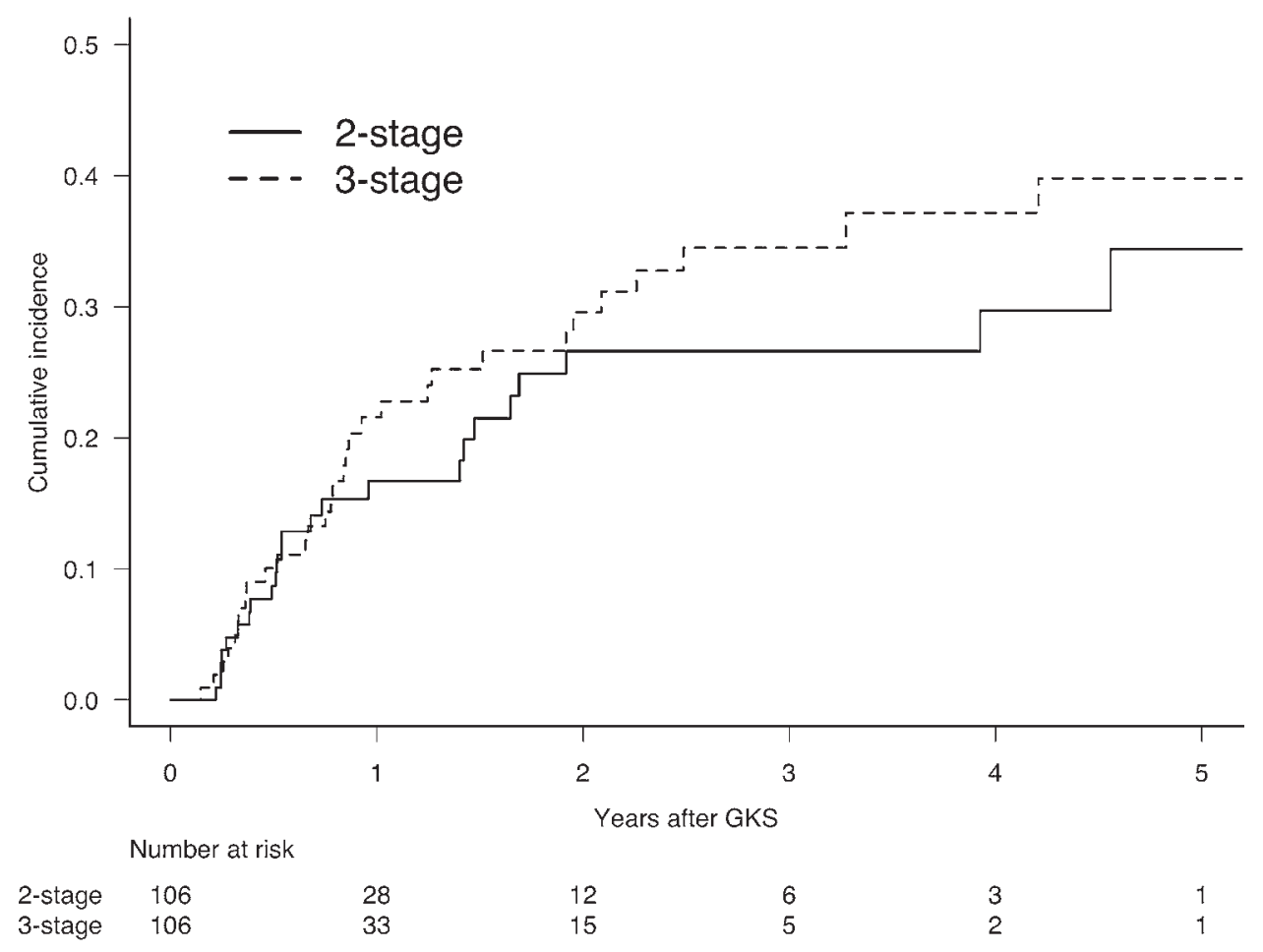

FIG. 3. Tumor progression: 3 -stage versus 2-stage GKS in the 212 case-matched patients. Cumulative incidences of tumor progression were slightly higher in the 3 -stage group than in the 2 -stage group ( $21.6 \%$ vs $16.7 \%$ at 1 year, respectively), but these differences did not reach statistical significance $(p=0.31)$. 
hypofractionated SRT. Although 3-stage GKS reduced tumor recurrence and radiation necrosis, it did not come into widespread use because the public health insurance in Japan did not support all costs, it was difficult to adjust treatment schedules for subsequent GKS procedures and chemotherapy, and repeated frame fixation was burdensome for patients. Since Yomo et al. reported on 2-stage GKS in 2012, which was a simplification of the 3-stage procedure, nearly half of the hospitals in Japan have applied 2-stage GKS in selected patients. ${ }^{45,46}$

In their in vivo study, Shibamoto et al. reported that the conversion of hypofractionated radiation doses to single doses using the linear quadratic (LQ) formula under-estimates the effects of hypofractionated radiation by $20 \%-$ $40 \%$ and that the discrepancy tends to increase as the fraction number increases. ${ }^{13,14,21,23,26,35,36}$ There has been controversy regarding which is better, 3 -stage or 2-stage GKS. Therefore, the JLGKS conducted the present retrospective multi-institutional study (JLGK1601, UMIN000022152) to compare treatment results between 3-stage and 2-stage GKS for selected patients meeting the study criteria.

\section{JLGK1601 Study Protocol}

The JLGK1601 study protocol had six major inclusion criteria, as noted above in Methods. Of these six criteria, four (tumor number, KPS, leptomeningeal dissemination, and newly diagnosed BMs) are the same as those in the JLGK0901 study. ${ }^{44}$ The other two inclusion criteria concern maximum tumor volume and CITV. A maximum tumor volume of $33.5 \mathrm{~cm}^{3}$ is equal to a mean tumor diameter of $4 \mathrm{~cm}$. This upper limit was based on the first 3-stage GKS procedure described by Higuchi et al.," ${ }^{12}$ although Yomo et al. ${ }^{45,46}$ treated very large tumors, one with a volume of $55.3 \mathrm{~cm}^{3}$, using 2-stage GKS. Angelov et al. ${ }^{1}$ and Dohm et al..$^{5}$ recently validated 2-stage GKS treatment. The maximum tumor volume in the former study of 33 cases was $31.3 \mathrm{~cm}^{3}$, which was equivalent to the maximum among our 335 cases. ${ }^{1}$ In contrast, the maximum tumor volume in Dohm et al.'s study of 54 cases was 60.9 $\mathrm{cm}^{3} .5,45,46$ We restricted the CITV to $\leq 50 \mathrm{~cm}^{3}$ because we anticipated that a CITV exceeding that volume might be dangerous given our long-term experience as members of the JLGKS. However, there are no reports concerning the upper limits of maximum tumor volume or CITV while employing staged GKS. Further clinical studies are needed to ascertain the upper limit in tumor size (i.e., $\geq 4 \mathrm{~cm}$ in diameter) as well as a CITV $>50 \mathrm{~cm}^{3}$ that can be safely treated using these staged GKS techniques.

The JLGK1601 study protocol also restricted the treatment interval and the prescription dose delivered in each GKS procedure. The treatment interval was based on the two milestone-protocol studies on 3-stage GKS by Higuchi et al. ${ }^{12}$ and 2 -stage GKS by Yomo et al..$^{45,46}$ In our protocol, the treatment interval could be no longer than 42 days based on the repopulation of malignant cells. The interval between procedures had to be at least 12 days to achieve tumor volume reduction. The prescription doses had to be within 9.0-11.0 Gy in the 3-stage group. The prescription dose for 3-stage GKS was originally $10 \mathrm{~Gy}$, as reported by Higuchi et al., ${ }^{12}$ but was raised or reduced by 1 Gy (10\%) in our study. In the 2-stage group, the prescription dose range was $11.8-14.2 \mathrm{~Gy}$, which is essentially equal to that in the 3-stage procedure, providing 51.3-69.3 Gy in the 3-stage GKS and 51.4-68.7 Gy in the 2-stage GKS as the biological equivalent dose (BED) to malignant cells $(\alpha / \beta$ $=10$ ) based on the LQ model. ${ }^{40}$ The BEDs $(\alpha / \beta=2)$ were 148.5-214.5 Gy in the 3-stage approach and 162.8-230.0 Gy in the 2-stage approach, although there has been debate on whether the LQ model is applicable to radiosurgery, as mentioned above. ${ }^{13,14,17,21,23,26}$ Thus, we compared results between 3-stage and 2-stage GKS in strictly selected patients treated according to the JLGK1601 study protocol.

\section{Which Is Better, 2-Stage or 3-Stage GKS?}

At first, we expected the treatment results, especially tumor control and radiation-related adverse events, of the 3 -stage GKS to be superior to those of the 2-stage GKS because an increased number of stages would yield better tumor control with less radiation necrosis, according to Shibamoto and colleagues' experimental studies. ${ }^{13,14,21,23,26}$ However, little is known regarding the clinical superiority of 3-stage over 2-stage GKS. Thus, we adjusted for patient characteristics and staged GKS parameters according to the aforementioned strict study protocol, though there may have been some biases between the two groups. We, therefore, conducted a case-matched study employing the propensity score matching method for nine clinical factors, i.e., age, sex, KPS, primary cancer site, extracranial disease status, neurological symptoms, brain tumor numbers, maximum tumor volume, and CITV, which are known to be predictors of both OS and tumor control. ${ }^{27}$ We thereby minimized selection biases. This form of statistical analysis is the optimal approach in a retrospective study.

However, our JLGK1601 study results did not show a superiority of 3-stage GKS over 2-stage GKS in terms of OS. Neither did tumor progression, neurological deterioration, neurological death, or radiation-related adverse events differ between the two approaches. We concluded that treatment with 2-stage GKS with 11.8-14.2 Gy as the prescription dose and 12-42 days as the interval could be expected to yield results essentially equal to those of 3 -stage GKS with 9.0-11.0 Gy as the prescription dose and at least 12 days between procedures with a total treatment interval of no more than 42 days. Considering the burdens on patients, the costs, and the total treatment interval, we recommend 2-stage GKS rather than 3-stage GKS. Treatment intervals and prescription doses varied minimally in the 3-stage group, though some variations were observed in the 2-stage GKS group (Table 4). As regards the interval for the 2-stage GKS procedure, the tumor volume reduction ratio was significantly larger in 135 patients $(61.1 \%)$ with a longer interval (21-42 days) than in the 86 patients (38.9\%) with a shorter interval (12-20 days). Therefore, we recommend an interval of at least 3 weeks for 2-stage GKS.

\section{Limitations of the JLGK1601 Study}

This study has several limitations. First, the number of collected cases, especially those receiving 3-stage GKS, was limited. As mentioned above, there has been a shift from 3-stage GKS to 2-stage GKS, making it difficult to collect an adequate number of 3 -stage cases. Another op- 
tion may be to involve Gamma Knife sites in countries outside of Japan to perform multicenter studies comparing 3-stage and 2-stage GKS. Second, this study did not include patients who withdrew for various reasons, e.g., rapid progression of extracranial or intracranial disease status, refusal to continue staged procedures, etc. On occasion, we encounter patients with unexpected edema or bleeding, necessitating discontinuation or postponement of the staged procedures. Strictly speaking, a prospective study with intention to treat is needed to draw a definite conclusion about which GKS procedure is better. Finally, most of the cases registered in this study were treated in the first decade of this century. At that time, we did not have enough information about driver gene mutations. During this period, there was a dramatic paradigm shift from cytotoxic agents to molecular targeting agents, especially in patients with driver gene mutations, e.g., epidermal growth factor receptor and anaplastic lymphoma kinase inhibitor in lung adenocarcinoma patients or human epidermal receptor 2 in breast cancer patients. Tyrosine kinase inhibitors (TKIs) have recently been recognized as one of the most effective treatments for lung adenocarcinoma patients with driver gene mutations. ${ }^{10}$ However, most of our patients were treated in the era before routine TKI use for such patients. Actually, only 19 patients ( 5 in the 3 -stage group and 14 in the 2-stage group) received TKIs after staged GKS. Since 2010, more sophisticated molecular targeting agents and novel immune checkpoint inhibitors have improved OS in BM patients, and the strategies for treating BMs have changed dramatically, although SRS is still the mainstay treatment for patients with several BMs causing neurological symptoms.

\section{Is Staged GKS a Reasonable Treatment for Unresectable BMs $<4 \mathrm{~cm}$ in Diameter?}

Surgery has long been the mainstay treatment for large BMs. ${ }^{28,29}$ However, staged GKS appears to be more beneficial than surgery in patients with BMs that are unresectable or occur in multiples. Furthermore, staged GKS has advantages over single-fraction SRS utilizing a palliative dose, especially in patients expected to have moderate or even prolonged survival, i.e., those who are classified as RPA class I or II, ${ }^{9}$ modified RPA class I+IIa or IIb, ${ }^{43}$ Graded Prognostic Assessment (GPA) score 3.0 or 3.54.0, ${ }^{37-39}$ Basic Score for Brain Metastases (BSBM) 2 or $3,{ }^{18}$ or Score Index for Radiosurgery (SIR) $4-7$ or $8-10,{ }^{42}$ making better BM control more necessary. Serizawa and colleagues proposed the neurological prognostic score (NPS) for estimating intracranial disease progression using four factors (number of brain tumors, CITV, leptomeningeal dissemination, and neurological symptoms). ${ }^{31,32}$ The NPS A has a better neurological prognosis, whereas the NPS B has a poorer one. This NPS can be incorporated into the aforementioned prognostic scores, enabling us to predict survival and determine the neurological prognosis simultaneously. Most patients in our study (282 patients [84.2\%]) were scored as NPS B. For the NPS B patients, especially those in good condition, it is crucial to choose more intensive radiation methods, i.e., staged GKS rather than palliative GKS, to minimize tumor recurrence and radiation necrosis. Worldwide, staged GKS appears to be difficult due to economic reasons as well as schedule adjustments between chemotherapy and subsequent GKS procedures. However, staged GKS may be considered appropriate for patients with an expectation of moderate to good survival.

The JLGK1601 study demonstrated acceptable tumor progression incidences $(21.0 \%$ in the 3-stage group and $20.7 \%$ in the 2 -stage group at 1 year) and low incidences of neurological deterioration (15.2\% vs $15.3 \%$ at 1 year) and neurological death $(4.8 \%$ vs $6.8 \%$ at 1 year). These rates were superior to those previously reported using fractionated SRT. 6,20,22,25,41 Furthermore, the 1-year incidences of CTCAE grade 3 or worse were acceptably low (3.7\% vs $3.5 \%$ at 1 year, respectively). Treatment results of 2 -stage GKS were not inferior to those of the 3-stage GKS, either in the main cohort of 335 patients or in the subset of 212 case-matched subjects. Thus, we can conclude that a 2-staged approach as well as a 3-staged approach would be an option for newly diagnosed, especially large (3-4 cm in diameter), unresectable BMs if the following criteria are met: no more than 10 lesions, no MRI findings of leptomeningeal dissemination, KPS $70 \%$ or better, and CITV of $50 \mathrm{~cm}^{3}$ or less. These staged protocols are also applicable to the mask system with ICON or other linear accelerator systems. In the future, randomized studies comparing these staged GKS procedures to other treatments, i.e., fractionated SRT, surgical removal, and WBRT, are needed.

\section{Conclusions}

Our JLGK1601 study demonstrated that staged GKS achieves good tumor control and prevents neurological deterioration and death with minimal radiation-related adverse events. Treatment results for 2 -stage GKS were the same as those for 3-stage GKS in the main cohort of 335 patients and in 212 case-matched patients. Three- and 2-stage GKS procedures are good treatment options for newly diagnosed, especially unresectable, BMs that are large $(3-4 \mathrm{~cm}$ in diameter) if the criteria of no more than 10 lesions, no leptomeningeal dissemination, a KPS $70 \%$ or better, and a CITV of $50 \mathrm{~cm}^{3}$ or less are met.

\section{Acknowledgments}

We are very grateful to all investigators of the JLGK1601 Study Group not included as co-authors, as well as Ms. Yukiko Hanawa, Tokyo Gamma Unit Center, Tsukiji Neurological Clinic, for her meticulous work in managing the database. We thank Bierta E. Barfod, Katsuta Hospital Mito GammaHouse, for her help with English editing.

\section{References}

1. Angelov L, Mohammadi AM, Bennett EE, Abbassy M, Elson P, Chao ST, et al: Impact of 2-staged stereotactic radiosurgery for treatment of brain metastases $\geq 2 \mathrm{~cm}$. J Neurosurg 129:366-382, 2018

2. Baschnagel AM, Meyer KD, Chen PY, Krauss DJ, Olson RE, Pieper DR, et al: Tumor volume as a predictor of survival and local control in patients with brain metastases treated with Gamma Knife surgery. J Neurosurg 119:1139-1144, 2013

3. Chernov M, Hayashi M, Izawa M, Ochiai T, Usukura M, Abe $\mathrm{K}$, et al: Differentiation of the radiation-induced necrosis and 
tumor recurrence after gamma knife radiosurgery for brain metastases: importance of multi-voxel proton MRS. Minim Invasive Neurosurg 48:228-234, 2005

4. Cummings M, Youn P, Bergsma DP, Usuki KY, Walter K, Sharma M, et al: Single-fraction radiosurgery using conservative doses for brain metastases: Durable responses in select primaries with limited toxicity. Neurosurgery [epub ahead of print], 2017

5. Dohm A, McTyre ER, Okoukoni C, Henson A, Cramer CK, LeCompte MC, et al: Staged stereotactic radiosurgery for large brain metastases: local control and clinical outcomes of a one-two punch technique. Neurosurgery [epub ahead of print], 2017

6. Eaton BR, Gebhardt B, Prabhu R, Shu HK, Curran WJ Jr, Crocker I: Hypofractionated radiosurgery for intact or resected brain metastases: defining the optimal dose and fractionation. Radiat Oncol 8:135, 2013

7. Ebner D, Rava P, Gorovets D, Cielo D, Hepel JT: Stereotactic radiosurgery for large brain metastases. J Clin Neurosci 22:1650-1654, 2015

8. Fine JP, Gray RJ: A proportional hazards model for the subdistribution of a competing risk. J Am Stat Assoc 94:496509, 1999

9. Gaspar L, Scott C, Rotman M, Asbell S, Phillips T, Wasserman T, et al: Recursive partitioning analysis (RPA) of prognostic factors in three Radiation Therapy Oncology Group (RTOG) brain metastases trials. Int J Radiat Oncol Biol Phys 37:745-751, 1997

10. Gerber NK, Yamada Y, Rimner A, Shi W, Riely GJ, Beal K, et al: Erlotinib versus radiation therapy for brain metastases in patients with EGFR-mutant lung adenocarcinoma. Int J Radiat Oncol Biol Phys 89:322-329, 2014

11. Gooley TA, Leisenring W, Crowley J, Storer BE: Estimation of failure probabilities in the presence of competing risks: new representations of old estimators. Stat Med 18:695-706, 1999

12. Higuchi Y, Serizawa T, Nagano O, Matsuda S, Ono J, Sato M, et al: Three-staged stereotactic radiotherapy without whole brain irradiation for large metastatic brain tumors. Int J Radiat Oncol Biol Phys 74:1543-1548, 2009

13. Iwata H, Matsufuji N, Toshito T, Akagi T, Otsuka S, Shibamoto Y: Compatibility of the repairable-conditionally repairable, multi-target and linear-quadratic models in converting hypofractionated radiation doses to single doses. J Radiat Res (Tokyo) 54:367-373, 2013

14. Iwata H, Shibamoto Y, Murata R, Tomita N, Ayakawa S, Ogino $\mathrm{H}$, et al: Estimation of errors associated with use of linear-quadratic formalism for evaluation of biologic equivalence between single and hypofractionated radiation doses: an in vitro study. Int J Radiat Oncol Biol Phys 75:482-488, 2009

15. Kano H, Kondziolka D, Lobato-Polo J, Zorro O, Flickinger JC, Lunsford LD: T1/T2 matching to differentiate tumor growth from radiation effects after stereotactic radiosurgery. Neurosurgery 66:486-492, 2010

16. Karnofsky DA, Burchenal JH: The clinical evaluation of chemotherapeutic agents in cancer, in MacLeod CM (ed): Evaluation of Chemotherapeutic Agents. New York: Columbia University Press, 1949, pp 191-205

17. Kirkpatrick JP, Brenner DJ, Orton CG: Point/Counterpoint. The linear-quadratic model is inappropriate to model high dose per fraction effects in radiosurgery. Med Phys 36:33813384, 2009

18. Lorenzoni J, Devriendt D, Massager N, David P, Ruíz S, Vanderlinden B, et al: Radiosurgery for treatment of brain metastases: estimation of patient eligibility using three stratification systems. Int J Radiat Oncol Biol Phys 60:218-224, 2004

19. Minamimoto R, Saginoya T, Kondo C, Tomura N, Ito K,
Matsuo Y, et al: Differentiation of brain tumor recurrence from post-radiotherapy necrosis with 11C-methionine PET: visual assessment versus quantitative assessment. PLoS One 10:e0132515, 2015

20. Minniti G, D'Angelillo RM, Scaringi C, Trodella LE, Clarke E, Matteucci P, et al: Fractionated stereotactic radiosurgery for patients with brain metastases. J Neurooncol 117:295301, 2014

21. Miyakawa A, Shibamoto Y, Otsuka S, Iwata H: Applicability of the linear-quadratic model to single and fractionated radiotherapy schedules: an experimental study. J Radiat Res (Tokyo) 55:451-454, 2014

22. Murai T, Ogino H, Manabe Y, Iwabuchi M, Okumura T, Matsushita Y, et al: Fractionated stereotactic radiotherapy using CyberKnife for the treatment of large brain metastases: a dose escalation study. Clin Oncol (R Coll Radiol) 26:151158,2014

23. Nagai A, Shibamoto Y, Yoshida M, Wakamatsu K, Kikuchi Y: Treatment of single or multiple brain metastases by hypofractionated stereotactic radiotherapy using helical tomotherapy. Int J Mol Sci 15:6910-6924, 2014

24. Oermann EK, Kress MA, Todd JV, Collins BT, Hoffman R, Chaudhry H, et al: The impact of radiosurgery fractionation and tumor radiobiology on the local control of brain metastases. J Neurosurg 119:1131-1138, 2013

25. Ogura K, Mizowaki T, Ogura M, Sakanaka K, Arakawa Y, Miyamoto S, et al: Outcomes of hypofractionated stereotactic radiotherapy for metastatic brain tumors with high risk factors. J Neurooncol 109:425-432, 2012

26. Otsuka S, Shibamoto Y, Iwata H, Murata R, Sugie C, Ito M, et al: Compatibility of the linear-quadratic formalism and biologically effective dose concept to high-dose-per-fraction irradiation in a murine tumor. Int J Radiat Oncol Biol Phys 81:1538-1543, 2011

27. Parsons LS: Reducing bias in a propensity score matched pair sample using greedy matching techniques. SAS. (http:// www2.sas.com/proceedings/sugi26/p214-26.pdf) [Accessed August 24, 2018]

28. Patchell RA, Tibbs PA, Regine WF, Dempsey RJ, Mohiuddin M, Kryscio RJ, et al: Postoperative radiotherapy in the treatment of single metastases to the brain: a randomized trial. JAMA 280:1485-1489, 1998

29. Patchell RA, Tibbs PA, Walsh JW, Dempsey RJ, Maruyama $\mathrm{Y}$, Kryscio RJ, et al: A randomized trial of surgery in the treatment of single metastases to the brain. $\mathbf{N}$ Engl J Med 322:494-500, 1990

30. Prabhu RS, Press RH, Patel KR, Boselli DM, Symanowski JT, Lankford SP, et al: Single-fraction stereotactic radiosurgery (SRS) alone versus surgical resection and SRS for large brain metastases: a multi-institutional analysis. Int J Radiat Oncol Biol Phys 99:459-467, 2017

31. Serizawa T, Higuchi Y, Nagano O, Matsuda S, Aoyagi K, Ono J, et al: Robustness of the neurological prognostic score in brain metastasis patients treated with Gamma Knife radiosurgery. J Neurosurg 127:1000-1006, 2017

32. Serizawa T, Higuchi Y, Nagano O, Matsuda S, Ono J, Saeki $\mathrm{N}$, et al: A new grading system focusing on neurological outcomes for brain metastases treated with stereotactic radiosurgery: the modified Basic Score for Brain Metastases. J Neurosurg 121 (Suppl):35-43, 2014

33. Serizawa T, Higuchi Y, Ono J, Matsuda S, Nagano O, Iwadate Y, et al: Gamma Knife surgery for metastatic brain tumors without prophylactic whole-brain radiotherapy: results in 1000 consecutive cases. J Neurosurg 105 (Suppl):86-90, 2006

34. Serizawa T, Saeki N, Higuchi Y, Ono J, Matsuda S, Sato $\mathrm{M}$, et al: Diagnostic value of thallium-201 chloride singlephoton emission computerized tomography in differentiating tumor recurrence from radiation injury after gamma knife 
surgery for metastatic brain tumors. J Neurosurg 102 (Suppl):266-271, 2005

35. Shibamoto Y, Miyakawa A, Otsuka S, Iwata H: Radiobiology of hypofractionated stereotactic radiotherapy: what are the optimal fractionation schedules? J Radiat Res (Tokyo) 57 (Suppl 1):i76-i82, 2016

36. Shibamoto Y, Otsuka S, Iwata H, Sugie C, Ogino H, Tomita $\mathrm{N}$ : Radiobiological evaluation of the radiation dose as used in high-precision radiotherapy: effect of prolonged delivery time and applicability of the linear-quadratic model. J Radiat Res (Tokyo) 53:1-9, 2012

37. Sperduto PW, Berkey B, Gaspar LE, Mehta M, Curran W: A new prognostic index and comparison to three other indices for patients with brain metastases: an analysis of $1,960 \mathrm{pa}-$ tients in the RTOG database. Int J Radiat Oncol Biol Phys 70:510-514, 2008

38. Sperduto PW, Kased N, Roberge D, Xu Z, Shanley R, Luo X, et al: Summary report on the graded prognostic assessment: an accurate and facile diagnosis-specific tool to estimate survival for patients with brain metastases. J Clin Oncol 30:419-425, 2012

39. Sperduto PW, Yang TJ, Beal K, Pan H, Brown PD, Bangdiwala $A$, et al: Estimating survival in patients with lung cancer and brain metastases: an update of the Graded Prognostic Assessment for Lung Cancer Using Molecular Markers (Lung-molGPA). JAMA Oncol 3:827-831, 2017

40. Tucker SL: Tests for the fit of the linear-quadratic model to radiation isoeffect data. Int J Radiat Oncol Biol Phys 10:1933-1939, 1984

41. Wegner RE, Leeman JE, Kabolizadeh P, Rwigema JC, Mintz $\mathrm{AH}$, Burton SA, et al: Fractionated stereotactic radiosurgery for large brain metastases. Am J Clin Oncol 38:135-139, 2015

42. Weltman E, Salvajoli JV, Brandt RA, de Morais Hanriot R, Prisco FE, Cruz JC, et al: Radiosurgery for brain metastases: a score index for predicting prognosis. Int J Radiat Oncol Biol Phys 46:1155-1161, 2000

43. Yamamoto M, Serizawa T, Sato Y, Kawabe T, Higuchi Y, Nagano O, et al: Validity of two recently-proposed prognostic grading indices for lung, gastro-intestinal, breast and renal cell cancer patients with radiosurgically-treated brain metastases. J Neurooncol 111:327-335, 2013
44. Yamamoto M, Serizawa T, Shuto T, Akabane A, Higuchi Y, Kawagishi J, et al: Stereotactic radiosurgery for patients with multiple brain metastases (JLGK0901): a multi-institutional prospective observational study. Lancet Oncol 15:387-395, 2014

45. Yomo S, Hayashi M: A minimally invasive treatment option for large metastatic brain tumors: long-term results of two-session Gamma Knife stereotactic radiosurgery. Radiat Oncol 9:132, 2014

46. Yomo S, Hayashi M, Nicholson C: A prospective pilot study of two-session Gamma Knife surgery for large metastatic brain tumors. J Neurooncol 109:159-165, 2012

\section{Disclosures}

The authors report no conflict of interest concerning the materials or methods used in this study or the findings specified in this paper.

\section{Author Contributions}

Conception and design: Serizawa, Higuchi, Yamamoto, Nagano, Sato. Acquisition of data: Serizawa, Yamamoto, Matsunaga, Nagano, Aoyagi, Yomo, Koiso, Hasegawa, Nakazaki, Moriki, Kondoh, Nagatomo, Okamoto, Kohda, Kawai, Shidoh, Shibazaki, Onoue, Kenai, Inoue, Mori. Analysis and interpretation of data: Serizawa, Higuchi, Yamamoto, Nagano, Sato. Drafting the article: Serizawa. Critically revising the article: Serizawa, Higuchi, Yamamoto, Nagano, Sato, Yomo, Hasegawa. Reviewed submitted version of manuscript: all authors. Approved the final version of the manuscript on behalf of all authors: Serizawa. Statistical analysis: Serizawa, Sato. Administrative/technical/material support: Higuchi. Study supervision: Higuchi, Yamamoto, Sato.

\section{Correspondence}

Toru Serizawa: Tokyo Gamma Unit Center, Tsukiji Neurological Clinic, Tokyo, Japan. gamma-knife.serizawa@nifty.com. 